08.3

\title{
Влияние интерфейсных эффектов на электронный спектр структур GaAs/AIGaAs, используемых для создания фотоприемных устройств среднего ИК-диапазона
}

\author{
() В.С. Кривобок ${ }^{1}$, Д.А. Пашкеев ${ }^{1,2}$, Д.А. Литвинов ${ }^{1}$, Л.Н. Григорьева ${ }^{1,3,9}$, С.А. Колосов ${ }^{1}$ \\ ${ }^{1}$ Физический институт им. П.Н. Лебедева РАН, Москва, Россия \\ ${ }^{2}$ НПО „Орион“, Москва, Россия \\ ${ }^{3}$ Московский государственный университет им. М.В. Ломоносова, Москва, Россия \\ ฯ E-mail: In.grigorjeva@physics.msu.ru
}

Поступило в Редакцию 22 ноября 2019г.

В окончательной редакции 22 ноября 2019г.

Принято к публикации 9 декабря 2019г.

\begin{abstract}
Продемонстрировано влияние переходных процессов, возникающих в ростовой камере установки молекулярно-пучковой эпитаксии, на структуру интерфейсов и электронный спектр квантовых ям $\mathrm{GaAs}_{\mathrm{Al}} \mathrm{Al}_{x} \mathrm{Ga}_{1-x} \mathrm{As}$, используемых для изготовления фотоприемных устройств (ФПУ) среднего ИК-диапазона. Показано, что такие процессы приводят к низкочастотному сдвигу рабочего перехода ФПУ, появлению в спектрах поглощения межзонного перехода, запрещенного правилами отбора, уменьшению энергетического сдвига между уровнями размерного квантования, сформированными легкими и тяжелыми дырками. Перечисленные эффекты обеспечивают простой подход для бесконтактной оценки качества интерфейсов в гетероструктурах $\mathrm{GaAs} / \mathrm{Al}_{x} \mathrm{Ga}_{1-x}$ As для ФПУ.
\end{abstract}

Ключевые слова: ИК-детектор, гетероструктура, квантовая яма, низкотемпературная фотолюминесценция.

DOI: 10.21883/PJTF.2020.06.49155.18130

Гетероструктуры $\mathrm{GaAs} / \mathrm{Al}_{x} \mathrm{Ga}_{1-x} \mathrm{As}$ с квантовыми ямами (КЯ) используются для создания фотоприемных устройств (ФПУ) среднего и дальнего ИК-диапазонов (в англоязычной литературе QWIP - quantum well infrared photodetector) (см., например, работы $[1,2]$ и приведенные в них ссылки). Обычно детектирование фотона происходит за счет перехода электрона с основного квантово-размерного уровня КЯ GaAs $\left(E_{1}\right)$ на первый возбужденный уровень $\left(E_{2}\right)$, расположенный вблизи дна зоны проводимости барьерного слоя, и дальнейшего его дрейфа во внешнем электрическом поле. Несмотря на довольно хорошо отработанную технологию изготовления, к настоящему времени изготовление QWIP, адаптированных под заданный спектральный диапазон, сопряжено с рядом технических сложностей. Они вызваны, в частности, влиянием реального профиля КЯ на электронную подсистему и соответственно на спектральную чувствительность. Эти сложности, особенно существенные при изготовлении гибридных QWIP с резонансной ближнепольной связью (см., например, $[3,4])$, сопряжены с фундаментальными проблемами, касающимися влияния интерфейсов на электронную подсистему КЯ [5].

В частности, даже при выращивании гетероструктур методом молекулярно-пучковой эпитаксии наблюдаются слабые искажения идеальных (резких) интерфейсов КЯ за счет инертности процессов, происходящих в ростовой камере и ячейках Кнудсена. В этом случае стандартная модель прямоугольной КЯ, которая обычно применяется для описания электронного спектра, вообще говоря, оказывается неприменимой. Соответственно изменение условий роста (скорость потоков, температура и др.) приводит к изменениям в характере переходных процессов и, как следствие, неконтролируемой модификации электронного спектра в номинально одинаковых структурах. Кроме того, искажение различается для верхнего и нижнего интерфейсов КЯ за счет разного характера переходных процессов при закрытии и открытии Al-ячейки. Это в свою очередь нарушает симметрию потенциала КЯ. Насколько нам известно, роль описанных выше интерфейсных эффектов при формировании электронного спектра и оптических свойств структур для QWIP в современной литературе не обсуждалась.

В настоящей работе сопоставление расчетов в рамках приближения огибающей волновой функции с результатами измерений спектров возбуждения фотолюминесценции используется для демонстрации изменений электронного спектра, вызванных размытием интерфейсов структур $\mathrm{GaAs} / \mathrm{Al}_{x} \mathrm{Ga}_{1-x} \mathrm{As}$ для QWIP. Показано, что ряд особенностей, наблюдаемых в спектрах возбуждения экситонной люминесценции, позволяет оценивать степень размытия двух интерфейсов КЯ и их отличие друг от друга.

Гетероструктуры $\mathrm{GaAs} / \mathrm{Al}_{x} \mathrm{Ga}_{1-x} \mathrm{As}$ с КЯ были выращены методом молекулярно-пучковой эпитаксии на полуизолирующих подложках $\mathrm{GaAs}$ (100) на установке Riber Epineat 3-5. Барьеры в гетероструктуре были образованы 31 слоем $\mathrm{GaAs} / \mathrm{Al}_{x} \mathrm{Ga}_{1-x} \mathrm{As}$ толщиной $50 \mathrm{~nm}$, ямы - 30 слоями GaAs толщиной $5.5 \mathrm{~nm}$. Легированные аналоги таких гетероструктур используются для изготовления ФПУ на спектральный диапазон 8-10 $\mu \mathrm{m}$. 


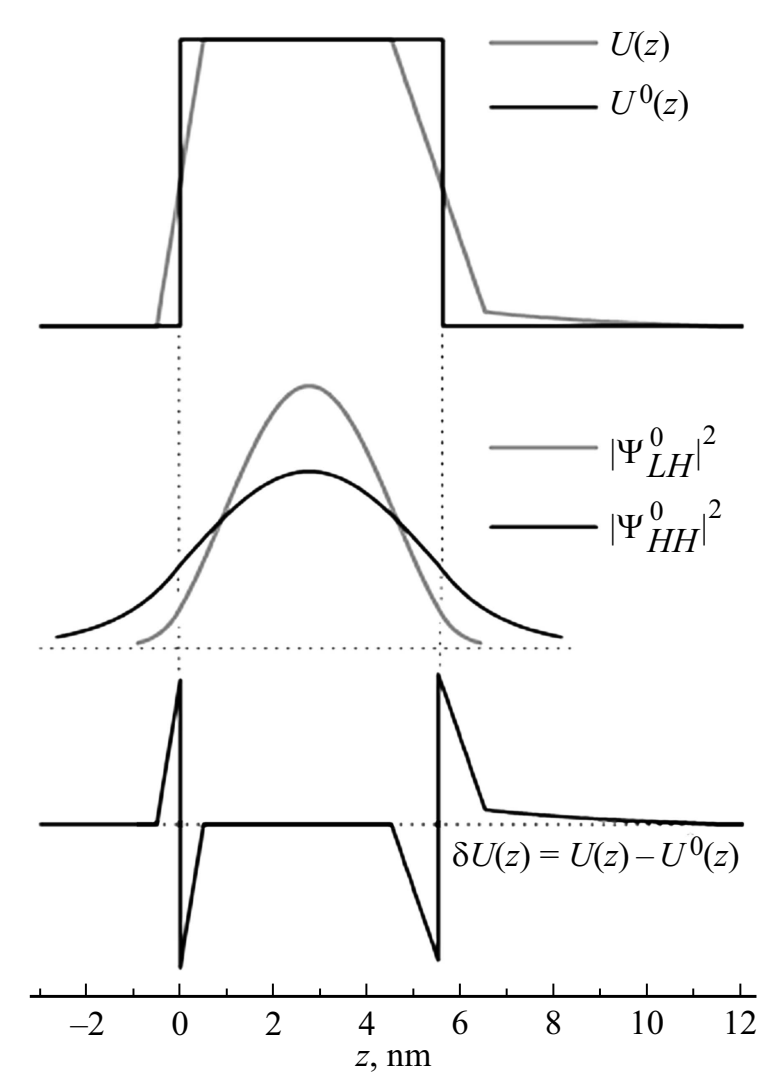

Рис. 1. Профили невозмущенного $U^{0}(z)$ и возмущенного $U(z)$ потенциалов, рассчитанные на основе ВЕР, структура невозмущенных волновых функций $\left|\Psi_{H H}^{0}\right|^{2}$ и $\left|\Psi_{L H}^{0}\right|^{2}$ для основных состояний, сформированных легкими и тяжелыми дырками в КЯ, и профиль возмущающего потенциала $\delta U(z)$, вызванного размытием интерфейсов КЯ.

Для испарения элементов третьей группы (Al, Ga) использовались эффузионные ячейки, для As - крекинговый источник. Для контроля температуры помимо использования термопар и пирометров проводились эллипсометрические измерения. Для наблюдения за ростом эпитаксиальных слоев использовалась дифракция быстрых электронов на отражение при скользящем падении. Качество структур независимо контролировалось с помощью рентгеноструктурного анализа. Для детальных исследований был отобран наиболее совершенный образец с нелегированными КЯ и мольным содержанием алюминия в барьерных слоях 21.8\%.

Поскольку рост КЯ GaAs/ $\mathrm{Al}_{x} \mathrm{Ga}_{1-x} \mathrm{As}$ идет в условиях стабилизации поверхности образца в потоках As, на размытие гетерограницы влияет только переключение материалов третьей группы $\mathrm{Ga}$ и $\mathrm{Al}$, участвующих в процессе роста соответствующего слоя. При открытии заслонки источника ему необходимо время, чтобы прийти в термодинамическое равновесие, поэтому соответствующее парциальное давление в пучке заметно меняется до выхода на стабильный режим. После закрытия заслонки вследствие временной задержки при откачке (удалении) остатков материала в камере также возникает посте- пенное изменение давления над образцом. Перед началом роста структуры данные изменения парциальных давлений в процессе открытия и закрытия заслонок источников измерялись при помощи ионизационного вакуумметра (ионизационная лампа помещалась над образцом). Предварительно калибровалось соответствие скоростей роста слоев определенному давлению потока материала (BEP - beam equivalent pressure, эквивалентное давление в атомарном пучке) при помощи измерений осцилляций рефлексов дифракционной картины быстрых электронов в процессе выращивания тестовых эпитаксиальных слоев при заданном давлении потоков материала. Из полученных данных и временных изменений давления над образцом рассчитывались форма и степень изменения состава тройного твердого раствора AlGaAs.

Для оценки изменений электронного спектра вследствие размытия интерфейсов на первом этапе разрывы валентной зоны и зоны проводимости аппроксимировались прямоугольной КЯ, для которой вычислялись огибающие волновых функций (ВФ) с использованием стандартных граничных условий Бастарда (рис. 1). В расчетах, детали которых описаны в [6], использовались широко распространенные параметры, доступные в известных справочных материалах [7]. В качестве примера на рис. 1 представлена структура ВФ $\left|\Psi_{H H}\right|^{2}$ и $\left|\Psi_{L H}\right|^{2}$ для двух нижних дырочных уровней в прямоугольной одномерной КЯ, соответствующей отобранному образу. Из рисунка видно, что на гетерогранице (у стенок КЯ) ВФ заметно затухают, поэтому поправку к потенциалу, вызванную размытием интерфейсов, можно оценивать в рамках теории возмущений. Соответственно ВФ, отвечающие резкому интерфейсу, рассматривались как невозмушенные.

На следующем этапе моделировались „реальные“ профили потенциала КЯ исходя из временны́х зависимостей ВЕР и литературных данных о зонной структуре $\mathrm{Al}_{x} \mathrm{Ga}_{1-x} \mathrm{As} / \mathrm{GaAs}$ [7]. Чтобы оценить профиль возмущающего потенциала $\delta U(z)$, мы использовали простое вычитание невозмущенного потенциала $U^{0}(z)$ из возмущенного $U(z)$. Профиль возмущения $\delta U(z)$ для дырок показан на рис. 1. Данное возмущение может несколько отличаться от результатов точных вычислений [5], но тем не менее правильно отражает качественный характер воздействия размытого интерфейса на электронно-дырочную систему КЯ. Аналогичная процедура была проведена для электронных состояний $E_{1}, E_{2}$, формируемых слоем КЯ в зоне проводимости.

Исходя из характера возмущения $\delta U(z)$ и структуры невозмущенных ВФ наряду с очевидным выталкиванием дырочных (электронных) уровней возмущение должно приводить к нескольким эффектам. Во-первых, за счет более сильного выталкивания электронного уровня $E_{1}$ по сравнению с выталкиванием первого возбужденного электронного уровня $E_{2}$ должен наблюдаться низкочастотный сдвиг рабочего перехода ФПУ, определяемый $\Delta E_{e}=E_{2}-E_{1}$. Данный эффект, характерный именно для 

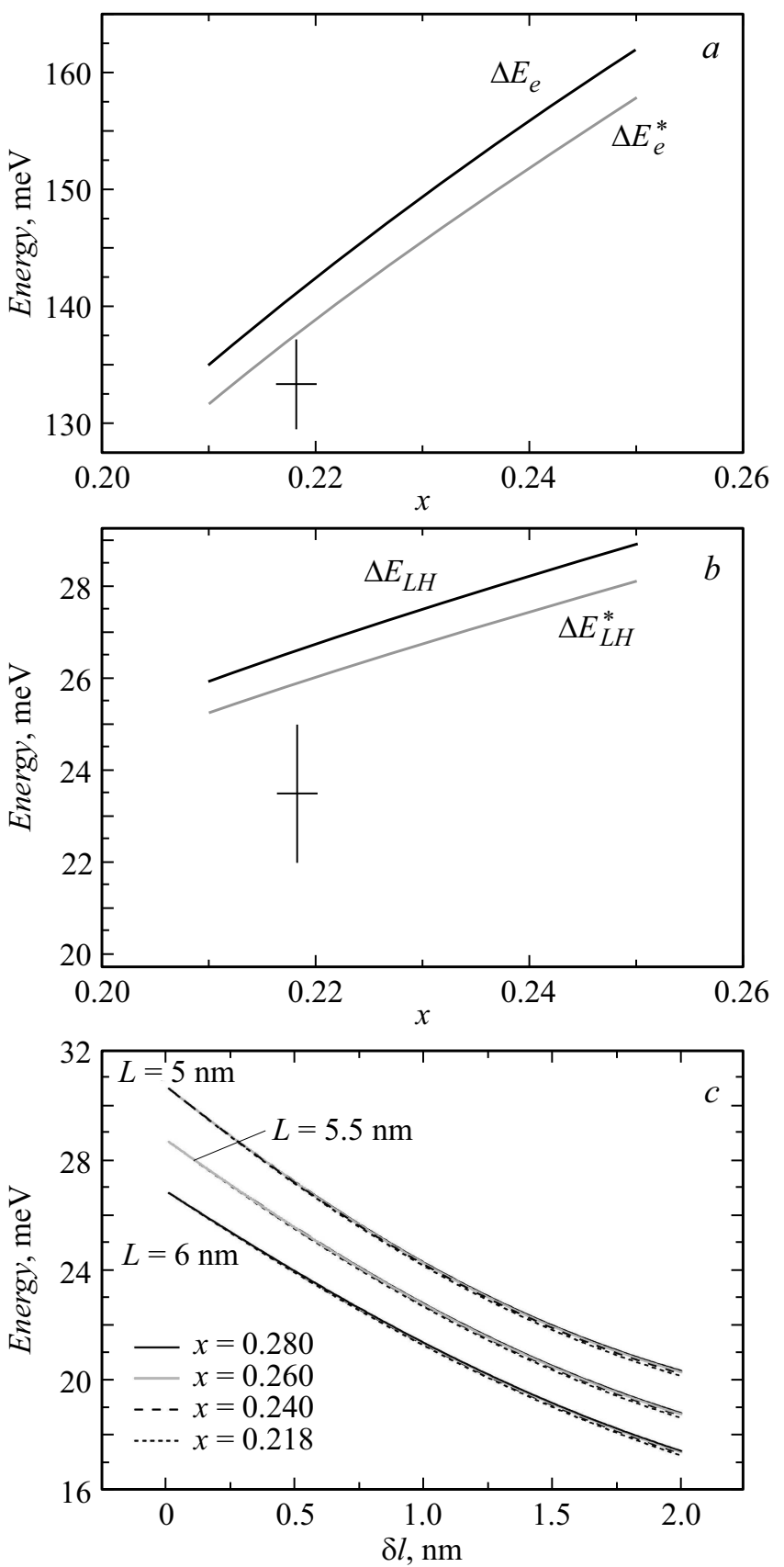

Рис. 2. $a-$ зависимости величины $\Delta E_{e}$, определяющей рабочий спектральный диапазон QWIP, от содержания Al в барьерных слоях $x$, рассчитанные без учета возмущения (черные кривые) и с его учетом (серые кривые). $b-$ результаты аналогичных расчетов для $\Delta E_{L H}$, определяющего энергетический сдвиг между двумя нижними дырочными уровнями в КЯ. $c$ - зависимость $\Delta E_{L H}$ от величины симметричного размытия $(\delta l)$ двух интерфейсов КЯ.

QWIP-структур, связан с тем, что ВФ для $E_{1}$ затухает вблизи интерфейса значительно быстрее, чем для $E_{2}$, ВФ которого заметно проникает в барьерные слои. Во-вторых, вследствие более медленного затухания ВФ $\left|\Psi_{L H}\right|^{2}$ вблизи интерфейсов следует ожидать уменьшения энергетического сдвига $\left(\Delta E_{L H}\right)$ между нижними уровнями размерного квантования, сформированными легкими и тяжелыми дырками. Наконец, отличие двух интерфейсов нарушает симметрию потенциала КЯ, сказываясь таким образом на правилах отбора для оптических переходов.

Рис. 2, $a$ иллюстрирует зависимости $\Delta E_{e}$ от содержания Al в барьерных слоях, рассчитанные без учета возмущения (черные кривые) и с его учетом (серые кривые). Можно заметить, что относительный сдвиг электронных уровней, вызванный оцененным выше размытием интерфейсов, достигает $5 \%$. На рис. $2, b$ показаны результаты аналогичных расчетов для $\Delta E_{L H}$. Как видно из рисунка, слабое размытие интерфейсов заметно уменьшает данную величину.

Для экспериментального определения $\Delta E_{e}, \Delta E_{L H}$ мы использовали спектры возбуждения экситонной фотолюминесценции (ФЛ) структур с нелегированными КЯ при низких (5 K) температурах. Рис. 3 иллюстрирует спектр возбуждения экситонной ФЛ для КЯ в отобранном образце. Ступенька, регистрируемая в высокоэнергетической части спектра, соответствует запрещенной зоне барьерных слоев $\mathrm{Al}_{x} \mathrm{Ga}_{1-x}$ As. Две особенности $E_{2}-H H_{1}$ и $E_{2}-H_{2}$ отвечают переходам между возбужденным электронным уровнем $E_{2}$ и основным $\left(H H_{1}\right)$ или первым возбужденным $\left(\mathrm{HH}_{2}\right)$ уровнями тяжелых дырок. Заметных признаков экситонных эффектов в данном случае не регистрируется. Следует отметить, что переход $E_{2}-H H_{1}$ является запрещенным для КЯ, имеющей симметричный профиль потенциала [8]. Его появление в спектре возбуждения ФЛ прямо указывает на отклонение профиля КЯ от симметричного. Сила осциллятора данного запрещенного перехода определяется различием двух интерфейсов КЯ.

Два интенсивных низкочастотных пика на рис. 3 отвечают экситонным резонансам с участием основного

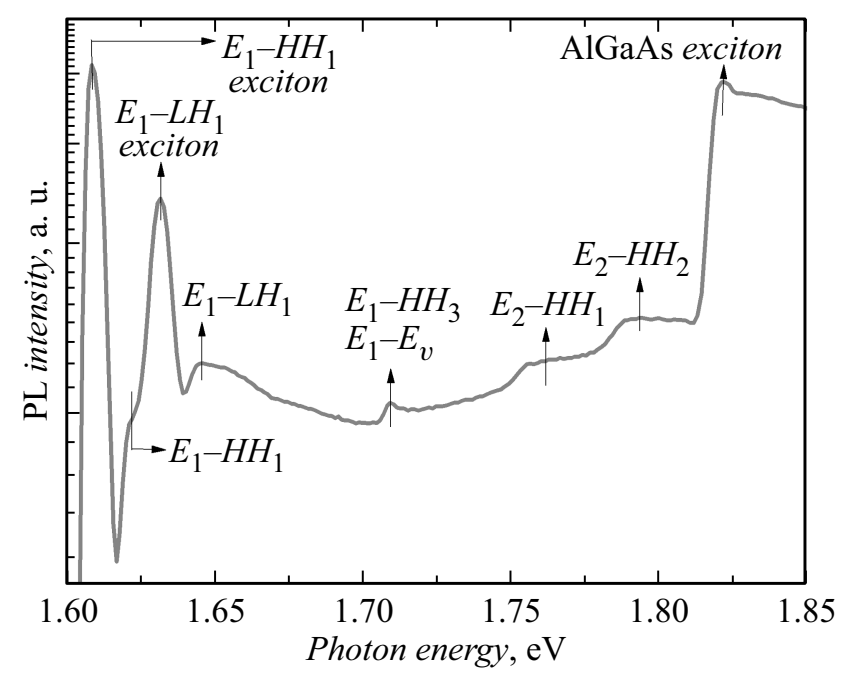

Рис. 3. Спектр возбуждения экситонной ФЛ для КЯ в отобранном образце, записанный при температуре $5 \mathrm{~K}$. 
электронного уровня $E_{1}$ и основного уровня легких $\left(L H_{1}\right)$ или тяжелых $\left(H H_{1}\right)$ дырок. Межзонные переходы $E_{1}-L H_{1}$ и $E_{1}-H H_{1}$, сформированные этими уровнями, проявляются в виде слабых ступенек, сдвинутых в высокоэнергетическую область по отношению к соответствующим экситонным резонансам. Наконец, особенность в районе $\sim 1.71 \mathrm{eV}$ отвечает переходам с участием $E_{1}$ и дырочных состояний, расположенных вблизи потолка валентной зоны барьерных слоев. Результаты расчета $\Delta E_{e}, \Delta E_{L H}$ на основе измеренного спектра возбуждения ФЛ показаны на рис. $2, a, b$ в виде крестов, отражающих погрешность эксперимента. Как видно из рисунка, учет размытия интерфейсов качественно объясняет низкочастотный сдвиг рабочего перехода QWIP $\left(\Delta E_{e}\right)$ и уменьшение расщепления между двумя верхними дырочными уровнями $\left(\Delta E_{L H}\right)$. Схожую тенденцию мы наблюдали и в случае других гетероструктур для QWIP или их нелегированных аналогов.

На рис. 2, $c$ приведены рассчитанные $\Delta E_{L H}$ в зависимости от величины симметричного размытия $(\delta l)$ для двух интерфейсов КЯ. Как видно из рисунка, $\Delta E_{L H}$, монотонно уменьшаясь с ростом $\delta l$, слабо зависит от содержания Al в барьерных слоях. Это означает, что $\Delta E_{L H}$ является прямым индикатором степени размытия интерфейсов при заданной ширине КЯ. Сделанный вывод качественно можно объяснить тем, что уровни $H H_{1}, L H_{1}$ в КЯ для QWIP резко затухают вблизи интерфейсов независимо от точных значений содержания Al в барьерных слоях и ширины КЯ. В то же время из-за разных эффективных масс, фигурирующих в граничных условиях Бастарда, значения $\left|\Psi_{H H}\right|^{2}$ и $\left|\Psi_{L H}\right|^{2}$ заметно различаются вблизи интерфейсов. Как следствие, различается и результат воздействия на них возмущением, связанным с размытым интерфейсом, но характер этого воздействия почти не меняется при небольших изменениях ширины КЯ и содержания Al в барьерах.

В целом полученные экспериментальные данные и результаты расчетов позволяют предложить достаточно простой метод для оценки качества интерфейсов в QWIP-гетероструктурах по спектрам возбуждения их экситонной ФЛ (или спектрам поглощения). В частности, для номинально одинаковых гетероструктур увеличение интенсивности запрещенного перехода $E_{2}-H H_{1}$ соответствует увеличению степени асимметрии потенциала КЯ. В то же время уменьшение энергетических сдвигов между электронными уровнями $E_{1}, E_{2}$ и дырочными уровнями $H H_{1}, L H_{1}$ является индикатором общего размытия интерфейсов КЯ. Следует отметить, что малые величины размытия интерфейсов, детектируемые описанным методом в номинально одинаковых структурах, довольно сложно контролировать с помощью электронной микроскопии и рентгеноструктурного анализа.

Дополнительные материалы к этой статье доступны в электронном виде (см. переводную версию).

\section{Финансирование работы}

Работа выполнена при поддержке Российского научного фонда (№ 19-79-30086).

\section{Конфликт интересов}

Авторы заявляют, что у них нет конфликта интересов.

\section{Список литературы}

[1] Rogalski A., Martyniuk P., Kopytko M. // Appl. Phys. Rev. 2017. V. 4. P. 031304.

[2] Gunapala S., Rhiger D., Jagadish C. // Advances in infrared photodetectors in semiconductors and semimetals. N.Y.: Academic Press, 2011. V. 84. 384 p.

[3] Luo L.B., Zeng L.H., Xie C., Yu Y.Q., Liang F.X., Wu C.Y., Wang L., Hu L.G. // Sci. Rep. 2014. V. 4. P. 3914.

[4] Hsu W.-C., Ling H.-S., Wang S.-Y., Lee C.-P. // Opt. Express. 2018. V. 26. P. 552-558.

[5] Rodina A.V., Alekseev A.Yu. // Phys. Rev. B. 2006. V. 73. P. 115312.

[6] Кривобок В.С., Литвинов Д.А., Николаев С.Н., Онищенко Е.Е., Пашкеев Д.А., Чернопицский М.А., Григорьева Л.Н. // ФТП. 2019. Т. 53. В. 12. С. 1632-1640.

[7] Semiconductors. Group IV elements, IV-IV and III-V compounds. Part b. Electronic, transport, optical and other properties / Eds O. Madelung, U. Rossler, M. Schulz. Landolt-Börnstein. Group III. Condensed matter. V. 41A1 $\beta$. Springer, 2002.

[8] Helm M. The basic physics of intersubband transitions // Semiconductors and semimetals / Eds R.K. Willardson, E.R. Beer. N.Y.: Academic Press, 1999. V. 62. P. 1-99. 